\title{
Alterações Hepáticas Causadas pelo Sars-CoV-2
}

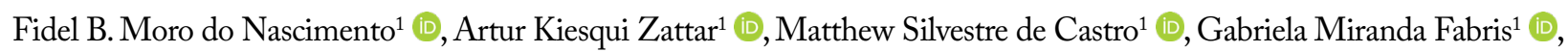

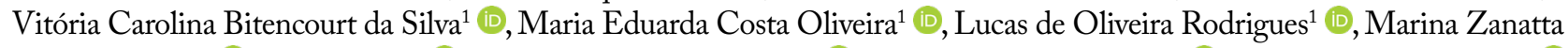

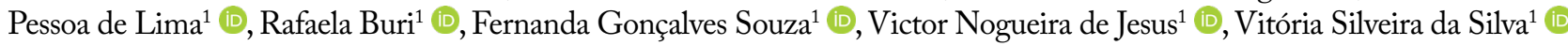

1. Centro Universitário de Várzea Grande Rór Várzea Grande (MT), Brasil.

doi https://doi.org/10.53855/bjt.v25i1.427_pt

Autor correspondente:

fidel.moro1@gmail.com

Editora de Seção

Ilka Boin

Recebido

Dez. 12, 2021

Aprovado

Jan. 27, 2022

Conflito de interesse

Nada a declarar.

\section{Como Citar}

Nascimento FBM, Zattar AK, Castro MS,

Fabris GM, Silva VCB, et al. Alterações

Hepáticas Causadas pelo Sars-CoV-2.

BJT.2022; 25(01): e0622. https://doi. org/10.53855/bjt.v25i1.427_pt

eISSN

2764-1589

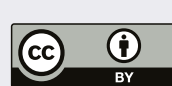

Resumo: Com a endemia do vírus Sars-CoV-2, muitos indivíduos acometidos de doenças hepáticas preexistentes como cirrose hepática e as hepatopatias crônicas se tornaram expostos à descompensação do quadro pela virulência da exposição e à suscetibilidade individual pela infecção do novo coronavírus. A citotoxicidade direta do vírus Sars-CoV-2 ocorre pela sua replicação nas células hepáticas, dada pela ligação do agente às células-alvo pela expressão da enzima conversora de angiotensina 2 (ECA2), que é a principal mediadora da replicação viral nos pacientes infectados pela Covid-19. Como consequência, as citocinas pró-inflamatórias aumentam e podem provocar hipóxia e isquemia sistêmica. Em associação à linfopenia e à diminuição dos níveis de células T-CD4+, os pacientes podem evoluir para descompensação ou piora do quadro infeccioso, com a insuficiência hepática crônica agudizada desde a primeira semana e, assim, diminuição de sobrevida. Os pacientes pré-diagnosticados com cirrose e infectados pelo vírus da Covid-19 possuem maior acometimento hepático e pior prognóstico e, portanto, merecem acompanhamento especial, sendo cuidadosamente avaliados a fim de possibilitar a redução das lesões hepáticas causadas pela infecção.

Descritores: Sars-CoV-2; Covid-19; Hepatócitos; Pandemias; Síndrome da liberação de citocina; Cirrose hepática; Hipóxia.

\section{INTRODUÇÃO}

A Covid-19 é uma infecção altamente contagiosa que surgiu como endemia em dezembro de 2019 com seus primeiros casos na província chinesa de Wuhan e em seguida evoluiu para uma pandemia. ${ }^{1}$ A alta taxa de transmissibilidade pelas vias interpessoal, contato com superfícies contaminadas e nosocomial é fator que justifica sua rápida disseminação mundial. ${ }^{2,3}$

Com o contágio pelo Sars-CoV-2, o vírus RNA de fita simples inicia a patogenicidade no indivíduo. O processo de invasão viral acontece por meio da ligação de grande afinidade da proteína S presente no vírus com a enzima conversora de angiotensina 2 (ECA2), a qual está altamente presente nas vias aéreas. Essa ligação viabiliza a penetração em células-alvo alveolares por meio do processo de endocitose dependente de clatrina, possibilitando assim a fusão e a replicação viral dentro das células alveolares e sua propagação para outros segmentos do pulmão e demais sistemas que expressem ECA2.,5 $\mathrm{O}$ acometimento sistêmico causado pela infecção por Sars-CoV-2 foi observado em células cardíacas, renais, neurológicas e hepáticas, esta última verificada em diversos estudos mediante a alteração de exames de proteínas marcadoras de lesão hepática. ${ }^{6-12}$

O quadro clínico da infecção viral é versátil, podendo ocorrer desde casos assintomáticose sintomáticos clássicos, como febre, tosse seca, fadiga, expectoração, dispneia, dor de garganta, cefaleia, mialgia ou artralgia, congestão nasal, diarreia, hemoptise e congestão conjuntival. ${ }^{13}$ 
A gravidade da infecção varia de acordo com a virulência da exposição e a suscetibilidade do indivíduo acometido. A classificação de fatores de risco para casos de maior gravidade do novo coronavírus são: idade avançada, obesidade, diabetes mellitus, hipertensão arterial sistêmica, doenças respiratórias crônicas, doenças cardiovasculares e neoplasias, evidenciando a importância das comorbidades nos pacientes. ${ }^{14}$

A lesão hepática é um importante marcador de agravamento da infecção nos indivíduos acometidos com a infecção pelo novo coronavírus, além de ser caracterizada como comorbidade em pacientes que já possuem doenças hepáticas preexistentes, como a cirrose hepática descompensada e as hepatopatias crônicas. ${ }^{15-17}$ Isso reflete diretamente não só no dano causado no fígado no momento da infecção, mas também no seguimento a longo prazo dos pacientes, como demonstra o estudo APCOLIS. ${ }^{18}$ Nesse trabalho sobre a evolução da doença hepática preexistente em pacientes com infecção por Sars-CoV-2, foi observada aceleração da lesão no fígado em portadores de cirrose quando comparado a indivíduos sem comorbidades. ${ }^{18}$

Este estudo realiza uma revisão não sistemática dos mecanismos de lesão hepática causados pelo Sars-CoV-2 e sua associação com o pré-diagnóstico de cirrose hepática, buscando analisar o agravamento da injúria preexistente do fígado, possíveis métodos de investigação laboratorial e prognósticos desses pacientes pós-infecção pela Covid-19.

\section{MECANISMOS DE LESÃO HEPÁTICA PELO SARS-COV-2}

Com o intuito de compreender como a Covid-19 pode afetar o fígado, um estudo de coorte foi realizado comparando diversos exames laboratoriais entre pacientes infectados que não apresentassem complicações hepáticas. ${ }^{19}$ Ainda que o mecanismo de lesão hepática pela Covid-19 não tenha sido completamente esclarecido, a investigação patológica de um paciente infectado demonstrou a presença de esteatose moderada, infiltração de leucócitos nas áreas lobulares e portal, necrose focal e congestionamento sinusoidal. ${ }^{19}$

\section{Citotoxicidade viral direta ao fígado}

A citotoxicidade direta por causa da replicação do vírus nas células hepáticas se deve pela ligação do Sars-CoV-2 às células-alvo, demonstrada pela expressão de ECA2, sendo a principal mediadora da replicação viral nos pacientes infectados pelo Sars-CoV-2. ${ }^{20}$

Um estudo realizado pelo Centro Universitário Médico de Groningen, na Holanda, analisou a distribuição da ECA2 nos tecidos corporais, detectando a presença dessas proteínas nos hepatócitos. ${ }^{21}$ Já recentemente, em 2020 , houve a publicação de um estudo de coorte que revelou enriquecimento significativo da expressão de ECA2 no fígado, principalmente nas células que compõem os ductos biliares (colangiócitos), observando uma média 20 vezes maior em relação ao nível de expressão em hepatócitos, enquanto nas células de Kupffer a ECA2 não foi detectada. ${ }^{22}$ Reforçando essa proposta, amostras de biópsia coletadas de pacientes com Covid-19 evidenciam esteatose microvesicular moderada e atividade lobular e portal leve. ${ }^{23}$

Pacientes infectados pelo vírus durante a hospitalização em Wuhan demonstraram aspartato aminotransferase (AST), alanina aminotransferase (ALT) e lactato desidrogenase (LDH) elevados, sugerindo lesão ligada à progressão do Sars-CoV-2. ${ }^{24}$

\section{Lesão hepática imunomediada pela resposta sistêmica do organismo}

Entre as possíveis explicações para tais processos está a lesão imunomediada pela resposta inflamatória sistêmica, conforme o relato de um estudo que evidenciou as citocinas pró-inflamatórias aumentadas principalmente em casos severos de Covid-19, o que poderia provocar hipóxia e isquemia sistêmica. ${ }^{25}$

O aumento exacerbado de citocinas inflamatórias foi descrito como tempestade de citocinas, e, em conjunto, a linfopenia e a diminuição dos níveis de células T-CD4+, achados comuns nos pacientes infectados pelo Sars-CoV-2, podem estar associadas à intensidade da doença e à mortalidade. ${ }^{26}$

Um dos estudos de coorte caracterizou, nas citocinas inflamatórias, a presença de alterações cinéticas, incluindo IL-6, IL-2, IL-4, IFN- $\gamma$ e TNF- $\alpha$ no soro dos participantes. Com isso, observou-se que em pacientes com características de infecção leve por Sars-CoV-2 as flutuações nos níveis séricos dessas citocinas eram consideravelmente menores do que em pacientes graves, os quais obtiveram flutuações significativas. ${ }^{27}$

Excetuando-se a IL-6, todas as outras citocinas atingiram seu pico no soro entre três e seis dias após o começo da doença, entretanto os níveis de IL-6 e IL-10 demonstraram aumento mantido no grupo com um quadro mais grave se comparado ao outro com uma infecção mais leve. Nessa lógica, viu-se que a diminuição das células T na Covid-19 pode resultar em agravamento das respostas inflamatórias, enquanto a normalização do número dessas células pode diminuir as respostas inflamatórias. Reforçando tal hipótese, a contagem de células $\mathrm{T}$ apontaram para uma correlação inversamente proporcional entre seus valores comparados aos níveis de citocinas no sangue periférico de pacientes graves, pois quando as células $\mathrm{T}$ estavam com os níveis mais baixos ocorreu o pico nos níveis séricos de IL-10, IL-2, IL-4, TNF- $\alpha$ e IFN- $\gamma$, aproximadamente entre o quarto e o sexto dia. ${ }^{27}$ 
Diante das pesquisas, observa-se um possível protagonismo da IL-6 no processo: 30,39\% dos participantes com infecção leve por Sars-CoV-2 tiveram os valores da IL-6 mais altos do que o normal, enquanto no grupo com infecção severa 76,19\% dos pacientes tiveram seu valor aumentado. Apesar de não completamente esclarecido, considera-se possível que tal processo ocorra em função da inibição das células Th2 participantes da imunidade humoral no estágio mais inicial da Covid-19. ${ }^{28}$

\section{Efeitos da hipoxemia grave no fígado}

Há três principais fatores de risco que geram hipoxemia grave nos pacientes que evoluem para acometimento hepático: insuficiência cardíaca, sepse grave e insuficiência respiratória. ${ }^{29,30}$ Nessas circunstâncias, uma tempestade de citocinas prejudica o adequado funcionamento do fígado, dificultando a transdução para sobrevivência celular, e provoca acúmulo significativo de lipídeos, como também o aumento do consumo de glicogênio e depleção de trifosfato de adenosina dos hepatócitos. Tais fatores associados causam estresse oxidativo e o aumento dos fatores pró-inflamatórios. ${ }^{27,31,32}$

Como consequência, os pacientes evoluem para um distúrbio circulatório decorrente de uma congestão passiva e da diminuição da perfusão hepática, o qual leva à hipóxia e isquemia. ${ }^{33}$ Com o dano hepático instalado, o edema intracelular da organela estará presente. Para reduzir tais efeitos negativos, as células de Kupffer aumentam a produção de citocinas para ativação dos leucócitos, o que promove aumento dos níveis de transaminase e lactato desidrogenase (LDH), gerando a necessidade de suporte de oxigênio. ${ }^{3}$

A Fig. 1 demonstra a atuação simultânea dos mecanismos de lesão hepática com a invasão do Sars-Cov-2 em hepatócitos e colangiócitos, acarretando disfunções irreversíveis, com consequente morte celular.

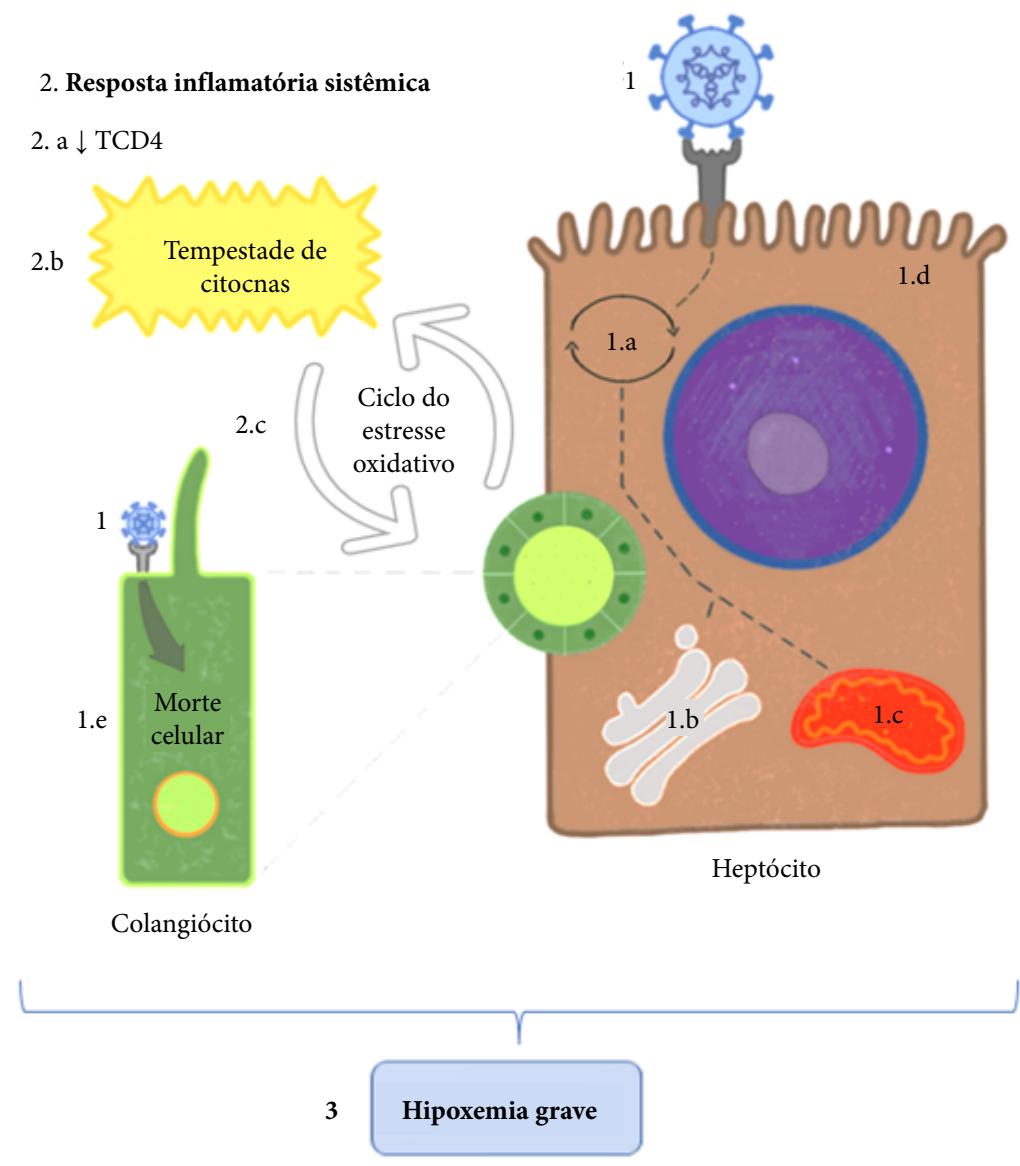

Figura 1. (1) O vírus Sars-CoV-2 adentra na célula por meio da ligação com a enzima conversora de angiotensina 2 (ECA2) expressa na superfície de hepatócitos e colangiócitos. (1.a) Replicação viral. (1.b) Estresse do retículo endoplasmático. (1.c) Edema mitocondrial. (1.d) Lesão de membrana celular. (1.e) Morte celular por conta dos danos irreversíveis às organelas. (2) Resposta inflamatória sistêmica. (2.a) Linfopenia caracterizada pela queda da contagem de linfócitos TCD4. (2.b) Aumento exacerbado de citocinas inflamatórias. (2.c) Ciclo do estresse oxidativo: o ambiente pró-inflamatório criado pelas citocinas dificulta o processo de respiração celular, lesando, desse modo, a célula hepática. Em consequência, há novo aumento dos fatores pró-inflamatório. (3) Hipoxemia grave, como resultado do distúrbio circulatório causado pelo ambiente inflamatório e células disfuncionais. 


\section{Evolução e prognóstico}

Pacientes em quadros graves de Covid-19 sem comorbidades hepáticas preexistentes demoram cerca de três semanas para começar a desenvolver lesões no fígado. Todavia, pacientes previamente cirróticos em estado compensado que são acometidos pela infecção possuem acelerada progressão de lesão hepática, podendo ser perceptível a descompensação ou a evolução da injúria com insuficiência hepática crônica agudizada desde a primeira semana. ${ }^{34,35}$

Nesses pacientes é possível observar aumento de citocinas pró-inflamatórias, associado com a presença de marcadores inflamatórios de lesão hepática, ponderado pelo aumento do nível das proteínas gama-glutamiltransferase, ALT e AST na corrente sanguínea. Essas proteínas foram dosadas como marcadores em estudos com pacientes cirróticos com Sars-CoV-2, além da presença de leucopenia, linfopenia e trombocitopenia nos exames laboratoriais. ${ }^{35-37}$

Por consequência do acometimento hepático causado pelo vírus da Covid-19, o paciente cirrótico controlado pode evoluir para um quadro de descompensação. Dessa forma, há maior dano ao fígado do paciente e diminuem-se as chances de sobrevida. ${ }^{38}$ Foi realizado um estudo baseado em exames cadavéricos de pacientes que vieram a óbito por cirrose descompensada e constatado que a invasão de células do fígado pelo vírus da Covid-19, associada com edema mitocondrial e lesão de membrana celular, causa, por conseguinte, a apoptose de hepatócitos, significativa inflamação focal lobular e infiltrado linfócito importante. ${ }^{39}$

A associação da cirrose hepática com o novo coronavírus foi relacionada com posições superiores de gravidade hepática na avaliação internacional representada pelo sistema Child-Pugh, com 1/3 dos pacientes sendo reclassificados em categorias mais severas. ${ }^{40}$ Os sistemas de avaliação do paciente hepatopata crônico são diretamente proporcionais à mortalidade pelo Sars-CoV-2. Ou seja, pacientes classificados como Child-Pugh altos possuem maiores chances de virem a óbito. ${ }^{41-43}$

Dessa maneira, é validado que pacientes pré-diagnosticados com cirrose e infectados pelo vírus da Covid-19 possuem maior acometimento hepático e pior prognóstico. ${ }^{37,38,40,41,43,46}$

\section{CONCLUSÃO}

Achados hepáticos são comuns na infecção pela Covid-19, ocasionados pela citotoxicidade direta, pela lesão imunomediada, por efeitos hipoxêmicos e, ainda, pelo próprio uso de medicamentos para tratamento, porém não houve confirmação precisa quanto aos efeitos diretos na morte de pacientes infectados pelo Sars-CoV-2 demonstrados em exames de autópsia. ${ }^{23}$ Pacientes com doenças hepáticas preexistentes merecem acompanhamento especial, já que as alterações clínicas do fígado se demonstram mais intensas do que o normal, representando um pior prognóstico e aumento da mortalidade em hepatopatas descompensados. ${ }^{40}$ Com base nas informações coletadas, o manejo e o tratamento da Covid-19 em pacientes devem ser cuidadosamente avaliados a fim de reduzir-se a lesão hepática causada pelo Sars-CoV-2.

\section{CONTRIBUIÇÃO DOS AUTORES}

Contribuições científicas e intelectuais substantivas para o estudo: Nascimento FBM; Fabris GM; Souza FG; Castro MS; Oliveira MEC; Silva VS e Buri R; Concepção e projeto: Nascimento FBM; Fabris GM; Souza FG; Castro MS; Oliveira MEC; Silva VS e Buri R; Análise e interpretação de dados: Silva VCB; Castro MS; Lima MKZ; Zattar AK; Jesus VN; Oliveira MEC; Silva VS; Rodrigues LO e Buri R; Escrita do manuscrito: Nascimento FBM; Fabris GM; Silva VCB; Castro MS; Lima MKZ; Zattar AK; Jesus VN; Oliveira MEC; Silva VS; Rodrigues LO e Buri R; Aprovação final: Nascimento FBM; Castro MS; Zattar AK; Oliveira MEC; Silva VS e Buri R.

\section{DISPONIBILIDADE DE DADOS DE PESQUISA}

Não se aplica.

\section{FINANCIAMENTO}

Não se aplica. 


\section{AGRADECIMENTOS}

Não se aplica.

\section{REFERÊNCIAS}

1. Zhu N, Zhang D, Wang W, Li X, Yang B, Song J, et al. A novel coronavirus from patients with pneumonia in China, 2019. New Eng J Med. 2020;382(8):727-33. https://doi.org/10.1056/NEJMoa2001017

2. Chan JFW, Yuan S, Kok KH, To KKW, Chu H, Yang J, et al. A familial cluster of pneumonia associated with the 2019 novel coronavirus indicating person-to-person transmission: a study of a family cluster. The Lancet. 2020;395(10223):514-23. https://doi.org/10.1016/s0140-6736(20)30154-9

3. Wang D, Hu B, Hu C, Zhu F, Liu X, Zhang J, et al. Clinical characteristics of 138 hospitalized patients with 2019 novel coronavirus-infected pneumonia in Wuhan, China. JAMA. 2020;323(11):1061-9. https://doi.org/10.1001/jama.2020.1585

4. Vaduganathan M, Vardeny P, Michel T, McMurray JJV, Pfeffer MA, Solomon SD. Renin-angiotensin-aldosterone system inhibitors in patients with Covid-19. N Engl J Med. 2020;382(17):1653-9. https://doi.org/10.1056/nejmsr2005760

5. Ye M, Wysocki J, William J, Soler MJ, Cokic I, Batlle D. Glomerular localization and expression of angiotensin-converting enzyme 2 and angiotensin-converting enzyme: Implications for albuminuria in diabetes. J Am Soc Nephrol. 2006;17(11):306775. https://doi.org/10.1681/asn.2006050423

6. Dweck MR, Bularga A, Hahn RT, Bing R, Lee KK, Chapman AR, et al. Global evaluation of echocardiography in patients with COVID-19. Eur Heart J Cardiovasc Imaging. 2020;21(9):949-58. https://oi.org/10.1093/ehjci/jeaa178

7. Cheng Y, Luo R, Wang K, Zhang M, Wang Z, Dong L, et al. Kidney disease is associated with in-hospital death of patients with COVID-19. Kidney Int. 2020;97(5):829-38. https://doi.org/10.1016/j.kint.2020.03.005

8. Mao L, Jin H, Wang M, Hu Y, Chen S, He Q, et al. Neurologic manifestations of hospitalized patients with coronavirus disease 2019 in Wuhan, China. JAMA Neurol. 2020;77(6):683-90. https://doi.org/10.1001/jamaneurol.2020.1127

9. Bernal-Monterde V, Casas-Deza D, Letona-Giménez L, Llama-Celis N de la, Calmarza P, Sierra-Gabarda O, et al. SARS-CoV-2 infection induces a dual response in liver function tests: Association with mortality during hospitalization. Biomedicines. 2020;8(9):328. https://doi.org/10.3390/biomedicines8090328

10. Guan W, Ni Z, Hu Y, Liang W, Ou C, He J, et al. Clinical characteristics of coronavirus disease 2019 in China. New Engl J Med. 2020;382(18):1708-20. https://doi.org/10.1056/NEJMoa2002032

11. Chen N, Zhou M, Dong X, Qu J, Gong F, Han Y, et al. Epidemiological and clinical characteristics of 99 cases of 2019 novel coronavirus pneumonia in Wuhan, China: a descriptive study. The Lancet. 2020;395(10223):507-13. https://doi.org/10.1016/s0140-6736(20)30211-7

12. Cai Q, Huang D, Yu H, Zhu Z, Xia Z, Su Y, et al. COVID-19: Abnormal liver function tests. J Hepatol. 2020;73(3):566-74. https://doi.org/10.1016/j.jhep.2020.04.006

13. World Health Organization. Mission China Joint. Report of the WHO-China Joint Mission on coronavirus disease 2019 (COVID-19). The WHO-China Joint Mission on Coronavirus Disease 2019. World Health Organization; 2020.

14. Galvão MHR, Roncalli AG. Factors associated with increased risk of death from covid-19: A survival analysis based on confirmed cases. Rev Bras Epidemiol. 2021;23:e200106. https://doi.org/10.1590/1980-549720200106

15. Bellentani S, Saccoccio G, Costa G, Tiribelli C, Manenti F, Sodde M, et al. Drinking habits as cofactors of risk for alcohol induced liver damage. Gut. 1997;41(6):845-50. https://doi.org/10.1136/gut.41.6.845

16. Poynard T, Bedossa P, Opolon P. Natural history of liver fibrosis progression in patients with chronic hepatitis C. The Lancet. 1997;349(9055):825-32. https://doi.org/10.1016/s0140-6736(96)07642-8

17. Farrell GC, Larter CZ. Nonalcoholic fatty liver disease: from steatosis to cirrhosis. Hepatology. 2006;43(2 Suppl. 1):S99-S112. https://doi.org/10.1002/hep.20973

18. Sarin SK, Choudhury A, Lau GK, Zheng MH, Ji D, Abd-Elsalam S, et al. Pre-existing liver disease is associated with poor outcome in patients with SARS CoV2 infection; The APCOLIS Study (APASL COVID-19 Liver Injury Spectrum Study). Hepatology Int. 2020;14(5):690-700. https://doi.org/10.1007/s12072-020-10072-8

19. Lei $\mathrm{P}$, Zhang L, Han P, Zheng C, Tong Q, Shang H, et al. Liver injury in patients with COVID-19: clinical profiles, CT findings, the correlation of the severity with liver injury. Hepatol Int. 2020;14(5):733-42. https://doi.org/10.1007/s12072-020-10087-1

20. Li W, Moore MJ, Vasilieva N, Sui J, Wong SK, Berne MA, et al. Angiotensin-converting enzyme 2 is a functional receptor for the SARS coronavirus. Nature. 2003;426(6965):450-4. https://doi.org/10.1038/nature02145

21. Hamming I, Timens W, Bulthuis MLC, Lely AT, Navis GJ, van Goor H. Tissue distribution of ACE2 protein, the functional receptor for SARS coronavirus. A first step in understanding SARS pathogenesis. J Pathol. 2004;203(2):631-7. https://doi. org/10.1002/path.1570 
22. Chai X, Hu L, Zhang Y, Han W, Lu Z, Ke A, et al. Specific ACE2 expression in cholangiocytes may cause liver damage after 2019-nCoV infection. BioRxiv. 2020. https://doi.org/10.1101/2020.02.03.931766

23. Xu Z, Shi L, Wang Y, Zhang J, Huang L, Zhang C, et al. Pathological findings of COVID-19 associated with acute respiratory distress syndrome. Lancet Respir Med. 2020;8(4):420-2. https://doi.org/10.1016/s2213-2600(20)30076-X

24. Wang D, Hu B, Hu C, Zhu F, Liu X, Zhang J, et al. Clinical characteristics of 138 hospitalized patients with 2019 novel coronavirus-infected pneumonia in Wuhan, China. JAMA. 2020;323(11):1061-9. https://doi.org/10.1001/jama.2020.1585

25. Wang Y, Liu S, Liu H, Li W, Lin F, Jiang L, et al. SARS-CoV-2 infection of the liver directly contributes to hepatic impairment in patients with COVID-19. J Hepatol. 2020;73(4):807-16. https://doi.org/10.1016\%2Fj.jhep.2020.05.002

26. Zhang C, Shi L, Wang FS. Liver injury in COVID-19: management and challenges. Lancet Gastroenterol Hepatol. 2020;5(5):428-30. https://doi.org/10.1016/s2468-1253(20)30057-1

27. Liu J, Li S, Liu J, Liang B, Wang X, Wang H, et al. Longitudinal characteristics of lymphocyte responses and cytokine profiles in the peripheral blood of SARS-CoV-2 infected patients. Ebiomedicine. 2020;55:102763. https://doi.org/10.1016/j.ebiom.2020.102763

28. Wan S, Yi Q, Fan S, Lv J, Zhang X, Guo L, et al. Characteristics of lymphocyte subsets and cytokines in peripheral blood of 123 hospitalized patients with2019novel coronaviruspneumonia(NCP).Medrxiv.https://doi.org/10.1101/2020.02.10.20021832

29. Birrer R, Takuda Y, Takara T. Hypoxic hepatopathy: pathophysiology and prognosis. Intern Med. 2007;46(14):1063-70. https://doi.org/10.2169/internalmedicine.46.0059

30. Wong CK, Lam CWK, Wu AKL, Ip WK, Lee NLS, Chan IHS, et al. Plasma inflammatory cytokines and chemokines in severe acute respiratory syndrome. Clin Exp Immunol. 2004;136(1):95-103. https://doi.org/10.1111/j.1365-2249.2004.02415.x

31. Kulkarni A, Kumar P, Tevethia HV, Premkumar M, Arab JP, Candia R, et al. Systematic review with meta-analysis: liver manifestations and outcomes in COVID-19. Aliment Pharmacol Ther. 2020;52(4):584-99. https://doi.org/10.1111/apt.15916

32. Bloom PP, Meyerowitz EA, Reinus Z, Daidone M, Gustafson J, Kim AY, et al. Liver Biochemistries in Hospitalized Patients With COVID-19. Hepatology. 2021;73(3):890-900. https://doi.org/10.1002/hep.31326

33. Varga Z, Flammer AJ, Steiger P, Haberecker M, Andermatt R, Zinkernagel AS, et al. Endothelial cell infection and endotheliitis in COVID-19. Lancet. 2020;395(10234):1417-8. https://doi.org/10.1016/s0140-6736(20)30937-5

34. Sarin SK, Choudhury A, Lau GK, Zheng M-H, Ji D, Abd-Elsalam S, et al. Pre-existing liver disease is associated with poor outcome in patients with SARS CoV2 infection; The APCOLIS Study (APASL COVID-19 Liver Injury Spectrum Study). Hepatol Int. 2020;14(5):690-700. https://doi.org/10.1007/s12072-020-10072-8

35. Ji D, Zhang D, Yang T, Mu J, Zhao P, Xu J, et al. Effect of COVID-19 on patients with compensated chronic liver diseases. Hepatol Int. 2020;14(5):701-10. https://doi.org/10.1007/s12072-020-10058-6

36. Marjani A, Mehrpouya M, Pourhashem Z. Serum $\gamma$-glutamyltransferase, alanine aminotransferase and aspartate aminotransferase activity in healthy blood donor of different ethnic groups in Gorgan. J Clin Diagn Res. 2016;10(7):BC04-6. https://doi.org/10.7860\%2FJCDR\%2F2016\%2F20919.8181

37. Melo APS, França EB, Malta DC, Garcia LP, Mooney M, Naghavi M. Mortalidade por cirrose, câncer hepático e transtornos devidos ao uso de álcool: Carga Global de Doenças no Brasil, 1990 e 2015. Rev Bras Epidemiol. 2017;20(Supl. 1):61-74. https://doi.org/10.1590/1980-5497201700050006

38. Kim D, Adeniji N, Latt N, Kumar S, Bloom PP, Aby ES, et al. Predictors of outcomes of COVID-19 in patients with chronic liver disease: US multi-center study. Clin Gastroenterol Hepatol. 2021;19(7):1469-79. https://doi.org/10.1016/j.cgh.2020.09.027

39. Rela M, Patil V, Narasimhan G, Jothimani D. COVID-19 in decompensated cirrhosis. Hepatol Int. 2020;14(6):1125-7. https:// doi.org/10.1007/s12072-020-10092-4

40. Qi X, Liu Y, Wang J, Fallowfield JA, Wang J, Li X, et al. Clinical course and risk factors for mortality of COVID-19 patients with pre-existing cirrhosis: A multicentre cohort study. Gut. 2021;70(2):433-6. https://doi.org/10.1136/gutjnl-2020-321666

41. Iavarone M, D’Ambrosio R, Soria A, Triolo M, Pugliese N, del Poggio P, et al. High rates of 30-day mortality in patients with cirrhosis and COVID-19. J Hepatol. 2020;73(5):1063-71. https://doi.org/10.1016/j.jhep.2020.06.001

42. Marjot T, Moon AM, Cook JA, Abd-Elsalam S, Aloman C, Armstrong MJ, et al. Outcomes following SARS-CoV-2 infection in patients with chronic liver disease: An international registry study. J Hepatol. 2021;74(3):567-77. https://doi.org/10.1016/j. jhep.2020.09.024

43. Qi X, Wang J, Li X, Wang Z, Liu Y, Yang H, et al. Clinical course of COVID-19 in patients with pre-existing decompensated cirrhosis: initial report from China. Hepatol Int. 2020;14(4):478-82. https://doi.org/10.1007/s12072-020-10051-z

44. Shalimar, Elhence A, Vaishnav M, Kumar R, Pathak P, Soni KD, et al. Poor outcomes in patients with cirrhosis and corona virus disease-19. Indian J Gastroenterol. 2020;39(3):285-91. https://doi.org/10.1007/s12664-020-01074-3

45. Bajaj JS, Garcia-Tsao G, Biggins SW, Kamath PS, Wong F, McGeorge S, et al. Comparison of mortality risk in patients with cirrhosis and COVID-19 compared with patients with cirrhosis alone and COVID-19 alone: multicentre matched cohort. Gut. 2021;70(3):531-6.

46. Hashemi N, Viveiros K, Redd WD, Zhou JC, McCarty TR, Bazarbashi AN, et al. Impact of chronic liver disease on outcomes of hospitalized patients with COVID-19: A multicentre United States experience. Liver Int. 2020;40(10):2515-21. https://doi.org/10.1111/liv.14583 